\title{
Stability of magnetic condensation and mass generation for confinement in SU(2) Yang-Mills theory
}

\author{
Kei-Ichi Kondo*i \\ Department of Physics, Faculty of Science, Chiba University, Chiba 263-8522, Japan \\ E-mail: kondokefaculty.chiba-u.jp
}

In the framework of the functional renormalization group, we reexamine the stability of the YangMills vacuum with a chromomagnetic condensation. We show that the Nielsen-Olesen instability of the Savvidy vacuum with a homogeneous chromomagnetic condensation disappears in the $S U$ (2) Yang-Mills theory. As a physical mechanism for maintaining the stability even for the small infrared cutoff, we argue that dynamical gluon mass generation occurs due to a BRSTinvariant vacuum condensate of mass dimension-two, which is related to two-gluon bound states identified with glueballs. These results support the dual superconductor picture for quark confinement.

From quarks and gluons to hadronic matter: A bridge too far?

2-6 September, 2013

European Centre for Theoretical Studies in Nuclear Physics and Related Areas (ECT*), Villazzano, Trento (Italy)

\footnotetext{
* Speaker.

${ }^{\dagger}$ This work is supported by Grant-in-Aid for Scientific Research (C) 24540252 from Japan Society for the Promotion of Science (JSPS).
} 


\section{Introduction}

The dual superconductor picture $[1]$ for the Yang-Mills theory vacuum is an attractive hypothesis for explaining quark confinement. The key ingredients of this picture are the existence of chromomagnetic monopole condensation and the dual Meissner effect. For the dual superconductor picture for the Yang-Mills theory vacuum to be true, the chromomagnetic monopole condensation must give a more stable vacuum than the perturbative one. In view of this, Savvidy [2] has argued based on the general analysis of the renormalization group equation that the dynamical generation of chromomagnetic field should occur in the Yang-Mills theory, i.e., a non-Abelian gauge theory with asymptotic freedom. Indeed, Savvidy has shown that the vacuum with nonvanishing homogeneous chromomagnetic field strength, i.e., the so-called Savvidy vacuum has lower energy density than the perturbative vacuum with zero chromomagnetic field. The effective potential $V(H)$ of the homogeneous chromomagnetic field $H$ has the form in $S U(2)$ Yang-Mills theory:

$$
V_{\text {Savvidy }}(H)=\frac{1}{2} H^{2}-\frac{\beta_{0} g^{2}}{16 \pi^{2}} \frac{1}{2} H^{2}\left(\ln \frac{g H}{\mu^{2}}+c\right), \quad \beta_{0}:=-\frac{22}{3}<0 .
$$

Then the effective potential $V(H)$ of the homogeneous chromomagnetic field $H$ has an absolute minimum at $H=H_{0} \neq 0$ away from $H=0$. The chromomagnetic condensation gives more stable vacuum than the perturbative one.

Immediately after his proposal, however, N.K.Nielsen and Olesen [3] have shown that the effective potential $V(H)$ of the homogeneous chromomagnetic field $H$, when calculated explicitly at one-loop level in the perturbation theory under the background gauge, develops a pure imaginary part:

$$
V_{\mathrm{NO}}(H)=\frac{1}{2} H^{2}-\frac{\beta_{0} g^{2}}{16 \pi^{2}} \frac{1}{2} H^{2}\left(\ln \frac{g H}{\mu^{2}}+c\right)+i \frac{g^{2} H^{2}}{8 \pi},
$$

in addition to the real part which agrees exactly with the Savvidy's result. This is called the NielsenOlesen (NO) instability of the Savvidy vacuum. The presence of the pure imaginary part implies that the Savvidy vacuum gets unstable due to gluon-antigluon pair annihilation.

This result is easily understood based on the following observation. In the homogeneous external chromomagnetic field $H$, the energy eigenvalue $E_{n}$ of the massless (off-diagonal) gluons with the spin $S=1\left(S_{z}= \pm 1\right)$ is given by

$$
E_{n}^{ \pm}=\sqrt{p_{\perp}^{2}+2 g H(n+1 / 2)+2 g H S_{z}}(n=0,1,2, \cdots),
$$

where $p_{\perp}$ denotes the momentum in those space-time directions which are not affected by the magnetic field and the index $n$ is a discrete quantum number which labels the Landau levels. Then the NO instability is understood as originating from the tachyon mode with $n=0$ and $S_{z}=-1$ (or the lowest Landau level for the gluon with spin one antiparallel to the external chromomagnetic field), since

$$
E_{0}^{-}=\sqrt{p_{\perp}^{2}-g H}
$$

becomes pure imaginary when $p_{\perp}^{2}<g H$. In other words, the NO instability of the Savvidy vacuum with homogeneous chromomagnetic condensation is due to the existence of the tachyon mode 
corresponding to the lowest Landau level which is realized by the applied external homogeneous chromomagnetic field.

A way to circumvent the NO instability is to introduce the magnetic domains (domain structure) with a finite extension into the vacuum [4]. The physical vacuum in Yang-Mills theory is split into an infinite number of domains with macroscopic extensions. Inside each such domain there is a nontrivial configuration of chromomagnetic field and the tachyon mode does not appear in the domain supporting $p_{\perp}^{2}>g H$. This resolution for the NO instability of Yang-Mills theory is called the Copenhagen vacuum or Spaghetti vacuum. It is instructive to recall that the vortices in a type II superconductor are neatly arranged into a hexagonal or occasionally square lattice.

The Copenhagen vacuum is a well-done model of the Yang-Mills vacuum. The domain structure introduces an infrared cutoff which prevents the momenta from taking the smaller values causing the instability. However, it is quite complicated to work out the dynamics of the Yang-Mills theory on the concrete inhomogeneous background. Therefore, there have been a lot of works trying to overcome the NO instability for the homogeneous chromomagnetic field.

In view of these, we reexamine the NO instability in the $S U(2)$ Yang-Mills theory in the framework of the functional renormalization group (FRG) [5] as a realization of the Wilsonian renormalization group. The FRG enables us to examine the effects caused by changing the infrared cutoff in a systematic way. In this paper we follow the methods developed for FRG in [6,7,010]. We point out the following results [11].

1. The Nielsen-Olesen instability in the effective potential $V(H)$ for the homogeneous chromomagnetic field $H$, i.e., the imaginary part $\operatorname{Im} V(H)$ of $V(H)$ disappears (or is absent from the beginning) in the framework of the FRG. (Therefore, the Nielsen-Olesen instability is an artifact of the one-loop calculation in the perturbation theory and it disappears in the non-perturbative framework beyond the perturbation theory.)

2. However, this result does not necessarily guarantee the automatic existence of the non-trivial homogeneous chromomagnetic field $H_{0} \neq 0$ as the minimum of the effective potential $V(H)$, such that $V\left(H_{0}\right)<V(H=0)=0$. (Therefore, the absence of the Nielsen-Olesen instability and the existence of the non-trivial minimum for the homogeneous chromomagnetic field in the effective potential are different problems to be considered independently.)

3. As a physical mechanism for maintaining the stability even for the small infrared cutoff, we propose the dynamical mass generation for the off-diagonal gluons (and off-diagonal ghosts), which is related to the BRST-invariant vacuum condensation of mass-dimension two [12, 13, 14, 15]. This gives a consistent picture compatible with the absence of the instability. (This leads to the Abelian dominance [16]: in the string tension extracted from the Wilson loop average and exponential-falloff of the off-diagonal gluon propagators $[17$, 18, 19] as well as the magnetic monopole dominance in the Maximal Abelian gauge.)

\section{Complex-valued flow equation in the FRG}

The effective average action $\Gamma_{\Lambda}$ with the infrared cutoff $\Lambda$ is obtained by solving the flow 
equation [5]:

$$
\partial_{t} \Gamma_{\Lambda}=\frac{1}{2} \operatorname{STr}\left[\left(\Gamma_{\Lambda}^{(2)}+R_{\Lambda}\right)^{-1} \cdot \partial_{t} R_{\Lambda}\right], \partial_{t}:=\Lambda \frac{d}{d \Lambda}
$$

where STr denotes the "supertrace" introduced for writing both commuting fields (e.g., gluons ) and anticommuting fields (e.g., quarks and the Faddeev-Popov ghosts), $R_{\Lambda}^{\Phi}$ is the infrared cutoff function for the field $\Phi$ which is introduced as the infrared regulator term in the form: $\int \Phi^{\dagger} R_{\Lambda}^{\Phi} \Phi$, and $\Gamma_{\Lambda}^{(2)}$ denotes the second functional derivatives of $\Gamma_{\Lambda}$ with respect to the field variables $\Phi$ :

$$
\left(\Gamma_{\Lambda}^{(2)}\right)_{\Phi^{\dagger} \Phi}=\frac{\vec{\delta}}{\delta \Phi^{\dagger}} \Gamma_{\Lambda} \frac{\overleftarrow{\delta}}{\delta \Phi}
$$

corresponding to the inverse exact propagator at the scale $\Lambda$. The ordinary effective action $\Gamma$ as the generating functional of the one-particle irreducible vertex functions is obtained in the limit $\Lambda \downarrow 0$ : $\Gamma=\lim _{\Lambda \downarrow 0} \Gamma_{\Lambda}$.

We consider the complex-valued effective average action $\Gamma_{\Lambda}=\Gamma_{\Lambda}^{\mathrm{R}}+i \Gamma_{\Lambda}^{\mathrm{I}}$ which is decomposed into the real part $\Gamma_{\Lambda}^{\mathrm{R}}:=\operatorname{Re} \Gamma_{\Lambda}$ and the imaginary part $\Gamma_{\Lambda}^{\mathrm{I}}:=\operatorname{Im} \Gamma_{\Lambda}$. Then it is shown (see Appendix A of [11]) that the flow equation is decomposed into two parts:

$$
\begin{aligned}
& \partial_{t} \Gamma_{\Lambda}^{\mathrm{R}}=\frac{1}{2} \operatorname{STr}\left\{\left[\left(\Gamma_{\Lambda}^{\mathrm{R}(2)}+R_{\Lambda}\right)^{2}+\left(\Gamma_{\Lambda}^{\mathrm{I}(2)}\right)^{2}\right]^{-1}\left(\Gamma_{\Lambda}^{\mathrm{R}(2)}+R_{\Lambda}\right) \partial_{t} R_{\Lambda}\right\}, \\
& \partial_{t} \Gamma_{\Lambda}^{\mathrm{I}}=-\frac{1}{2} \mathrm{~S} \operatorname{Tr}\left\{\left[\left(\Gamma_{\Lambda}^{\mathrm{R}(2)}+R_{\Lambda}\right)^{2}+\left(\Gamma_{\Lambda}^{\mathrm{I}(2)}\right)^{2}\right]^{-1} \Gamma_{\Lambda}^{\mathrm{I}(2)} \partial_{t} R_{\Lambda}\right\} .
\end{aligned}
$$

We find that the identically vanishing imaginary part $\Gamma_{\Lambda}^{\mathrm{I}}:=\operatorname{Im} \Gamma_{\Lambda} \equiv 0$ is a solution corresponding to a fixed point:

$$
\operatorname{Im} \Gamma_{\Lambda} \equiv 0 \text { for any value of } \Lambda \text {, }
$$

in sharp contrast with the real part. If $\Gamma_{\Lambda}^{\mathrm{I}} \neq 0$ for a certain value of $\Lambda$, it does not maintain the same value, i.e., $\beta\left(\Gamma_{\Lambda}^{\mathrm{I}}\right) \neq 0$. Thus the problem of showing the absence of the imaginary part $\operatorname{Im} \Gamma=\lim _{\Lambda \downarrow 0} \operatorname{Im} \Gamma_{\Lambda}$ in the effective action $\Gamma=\lim _{\Lambda \downarrow 0} \Gamma_{\Lambda}$ is reduced to proving the vanishing of the imaginary part $\operatorname{Im} \Gamma_{\Lambda}$ in the effective average action $\Gamma_{\Lambda}$ for a sufficiently large value of $\Lambda$ :

$$
\operatorname{Im} \Gamma_{\Lambda}=0 \text { for a certain value of } \Lambda \gg 1 .
$$

When $\Gamma_{\Lambda}^{\mathrm{I}}=0$, the flow equation for $\Gamma_{\Lambda}^{\mathrm{R}}$ turns into the standard flow equation.

\section{Flow equation in the chromomagnetic background}

We consider the $D$-dimensional Euclidean Yang-Mills theory. We decompose the $S U(2)$ YangMills field $\mathscr{A}_{\mu}=\mathscr{A}_{\mu}^{A} T^{A}$ into the background field $\mathscr{V}_{\mu}=\mathscr{V}_{\mu}^{A} T^{A}$ and the quantum fluctuation field $\mathscr{X}_{\mu}=\mathscr{X}_{\mu}^{A} T^{A}$ where $T^{A}=\frac{1}{2} \sigma^{A}$ with $\sigma^{A}$ being the Pauli matrices $(A=1,2,3)$ :

$$
\mathscr{A}_{\mu}^{A}=\mathscr{V}_{\mu}^{A}+\mathscr{X}_{\mu}^{A} \quad(A=1,2,3) .
$$

We can choose without loss of generality the diagonal field $V_{\mu}$ as the background field:

$$
\mathscr{V}_{\mu}^{A}(x)=\delta^{A 3} V_{\mu}(x)
$$


and the off-diagonal field $A_{\mu}^{a}(a=1,2)$ as the quantum fluctuation field:

$$
\mathscr{X}_{\mu}^{A}(x)=\delta^{A a} A_{\mu}^{a}(x), \quad(a=1,2) .
$$

In what follows, we prepare the diagonal field $V_{\mu}(x)$ of the form:

$$
V_{\mu}(x)=\frac{1}{2} x_{v} H_{v \mu}
$$

so that the $x$-independent homogeneous background field strength is realized:

$$
\mathscr{F}_{\mu \nu}^{A}[\mathscr{V}](x):=\partial_{\mu} \mathscr{V}_{\nu}^{A}(x)-\partial_{\nu} \mathscr{V}_{\mu}^{A}(x)+\varepsilon^{A B C} \mathscr{V}_{\mu}^{B}(x) \mathscr{V}_{v}^{C}(x)=\delta^{A 3}\left(\partial_{\mu} V_{v}(x)-\partial_{v} V_{\mu}(x)\right)=\delta^{A 3} H_{\mu \nu}
$$

The total effective average action $\Gamma_{\Lambda}$ is specified by giving the gauge-invariant part $\Gamma_{\Lambda}^{\text {inv }}$, the gauge-fixing (GF) part $\Gamma_{\Lambda}^{\mathrm{GF}}$ and the associated Faddeev-Popov (FP) ghost part $\Gamma_{\Lambda}^{\mathrm{FP}}$ :

$$
\Gamma_{\Lambda}=\Gamma_{\Lambda}^{\mathrm{inv}}+\Gamma_{\Lambda}^{\mathrm{GF}}+\Gamma_{\Lambda}^{\mathrm{FP}}
$$

We choose the background gauge as the gauge fixing condition to maintain the gauge invariance for the background field. In the above choice for the background field (3.2), the background gauge reduces to the maximal Abelian (MA) gauge:

$$
F^{a}:=\mathscr{D}_{\mu}^{a b}[V] A_{\mu}^{b}=0, \quad \mathscr{D}_{\mu}^{a b}[V]:=\partial_{\mu} \delta^{a b}-g \varepsilon^{a b 3} V_{\mu} .
$$

Then the gauge-fixing term is given by

$$
\Gamma^{\mathrm{GF}}=\int d^{D} \times \frac{1}{2 \alpha}\left(\mathscr{D}_{\mu}^{a b}[V] A_{\mu}^{b}\right)^{2},
$$

where $\alpha$ denotes the gauge-fixing parameter.

The FP ghost term is determined according to the standard procedure (see e.g., [20]) as

$$
\Gamma^{\mathrm{FP}}=\int d^{D} x\left\{i \bar{C}^{a} \mathscr{D}_{\mu}^{a b}[V] \mathscr{D}_{\mu}^{b c}[V] C^{c}-g^{2} \varepsilon^{a b 3} \varepsilon^{c d 3} i \bar{C}^{a} C^{d} A_{\mu}^{b} A_{\mu}^{c}+i \bar{C}^{a} g \varepsilon^{a b 3}\left(\mathscr{D}_{\mu}^{b c}[V] A_{\mu}^{c}\right) C^{3}\right\} .
$$

For the gauge-invariant part $\Gamma_{\Lambda}^{\text {inv }}$, we adopt the ansatz, a function $W_{\Lambda}$ of the gauge-invariant term $\Theta$ constructed from the field strength $\mathscr{F}_{\mu \nu}^{A}[\mathscr{A}]:=\partial_{\mu} \mathscr{A}_{v}^{A}-\partial_{v} \mathscr{A}_{\mu}^{A}+\varepsilon^{A B C} \mathscr{A}_{\mu}^{B} \mathscr{A}_{v}^{C}$ :

$$
\Gamma_{\Lambda}^{\mathrm{inv}}=\int d^{D} x W_{\Lambda}(\Theta(x)), \quad \Theta:=\frac{1}{4}\left(\mathscr{F}_{\mu v}^{A}[\mathscr{A}]\right)^{2} .
$$

$\Theta$ is decomposed as [21]

$$
\begin{gathered}
\Theta=\frac{1}{4}\left(\mathscr{F}_{\mu \nu}^{A}[\mathscr{V}]\right)^{2}+\frac{1}{2} A^{\mu a}\left(Q_{\mu \nu}^{a b}+\mathscr{D}_{\mu}^{a c}[V] \mathscr{D}_{\nu}^{c b}[V]\right) A^{v b}+\frac{1}{4}\left(\varepsilon^{3 a b} A_{\mu}^{a} A_{v}^{b}\right)^{2}, \\
Q_{\mu \nu}^{a b}:=-\left(\mathscr{D}^{2}\right)^{a b} \delta_{\mu \nu}+2 g \varepsilon^{a b} H_{\mu \nu}, \quad\left(\mathscr{D}^{2}\right)^{a b}:=\mathscr{D}_{\rho}^{a c}[V] \mathscr{D}_{\rho}^{c b}[V] .
\end{gathered}
$$

In the vanishing off-diagonal field limit $A_{\mu}^{a} \rightarrow 0, \Theta$ is reduced to

$$
\left.\Theta\right|_{A=0}=\frac{1}{4}\left(\mathscr{F}_{\mu v}^{A}[\mathscr{V}](x)\right)^{2}=\frac{1}{4}\left(\partial_{\mu} V_{v}(x)-\partial_{\nu} V_{\mu}(x)\right)^{2}=\frac{1}{2} H^{2},
$$


where $H:=\sqrt{\boldsymbol{H}^{2}}=\sqrt{\frac{1}{2} H_{\alpha \beta} H_{\alpha \beta}}>0$.

The off-diagonal gluon fields $A_{\mu}^{a}$ (and off-diagonal ghost fields $C^{a}, \bar{C}^{a}$ ) should be integrated out in the framework of the FRG following the idea of the Wilsonian renormalization group. For this purpose, we introduce the infrared regulator term $\Delta S_{\Lambda}$ for the off-diagonal gluon $A_{\mu}^{a}$ and off-diagonal ghosts $C^{a}, \bar{C}^{a}$ by

$$
\Delta S_{\Lambda}=\int_{p}\left[\frac{1}{2} A_{\mu}^{a}(p) R_{\Lambda, \mu v}\left(p^{2}\right) \delta^{a b} A_{v}^{b}(p)+\bar{C}^{a}(p) R_{\Lambda}\left(p^{2}\right) \delta^{a b} C^{b}(-p)\right](a, b=1,2),
$$

where $\int_{p}:=\int \frac{d^{D} p}{(2 \pi)^{D}}$ denotes the integration over the $D$-dimensional momentum space. We choose the infrared cutoff function with the structure:

$$
R_{\Lambda, \mu v}\left(p^{2}\right)=\delta_{\mu v} R_{\Lambda}\left(p^{2}\right)
$$

We adopt the proper-time form of the flow equation $[8]$ :

$$
\partial_{t} \Gamma_{\Lambda}=\int_{0}^{\infty} d \tau \frac{1}{2} \operatorname{STr}\left[e^{-\tau\left(\Gamma_{\Lambda}^{(2)}+R_{\Lambda}\right)} \partial_{t} R_{\Lambda}\right] .
$$

After performing the mode decomposition according to the projection method [6, 0, 10], the flow equation reads

$$
\begin{aligned}
\partial_{t} \Gamma_{\Lambda}= & \frac{1}{2} \int_{0}^{\infty} d \tau \Omega^{-1} \operatorname{Tr}\left[e^{-\tau\left(W_{\Lambda}^{\prime} Q+R_{\Lambda}^{\text {gluon }}\right)} \cdot \partial_{t} R_{\Lambda}^{\text {gluon }}\right] \text { (transverse gluons) } \\
& -\frac{1}{2} \int_{0}^{\infty} d \tau \Omega^{-1} \operatorname{Tr}\left[e^{-\tau\left(-W_{\Lambda}^{\prime} \mathscr{D}^{2}+R_{\Lambda}^{\text {gluon }}\right)} \cdot \partial_{t} R_{\Lambda}^{\text {gluon }}\right] \text { (scalar gluon) } \\
& +\frac{1}{2} \int_{0}^{\infty} d \tau \Omega^{-1} \operatorname{Tr}\left[e^{-\tau\left(-\alpha_{\Lambda}^{-1} \mathscr{D}^{2}+R_{\Lambda}^{\text {gluon }}\right)} \cdot \partial_{t} R_{\Lambda}^{\text {gluon }}\right] \text { (longitudinal gluon) } \\
& -\int_{0}^{\infty} d \tau \Omega^{-1} \operatorname{Tr}\left[e^{-\tau\left(-\tilde{Z}_{\Lambda} \mathscr{D}^{2}+R_{\Lambda}^{\text {ghost }}\right)} \cdot \partial_{t} R_{\Lambda}^{\text {ghost }}\right] \text { (ghosts ) }
\end{aligned}
$$

The spectrum sum is obtained from eigenvalues of the respective operator. The covariant Laplacian $-\left(\mathscr{D}_{\rho}[\mathscr{V}]\right)^{2}$ with the background field $\mathscr{V}$ which gives the (covariant constant) uniform chromomagnetic field $H$ has the spectrum:

$$
\operatorname{Spect}\left[-\mathscr{D}_{\rho}^{2}[\mathscr{V}]\right]=p_{\perp}^{2}+(2 n+1) g H,(n=0,1, \cdots),
$$

where $p_{\perp}$ denotes the $(D-2)$ dimensional (Fourier) momentum in those space-time directions which are not affected by the magnetic field (say, orthogonal to $1-2$ plane) and the index $n$ is a discrete quantum number which labels the Landau levels. We take into account the fact that the density of states is $\frac{g H}{2 \pi}$ for the Landau levels.

Moreover, the operator $Q_{\mu v}^{a b}$ with the same background field $\mathscr{V}$ has the spectrum:

$$
\operatorname{Spect}\left[Q_{\mu v}^{a b}\right]=\left\{\begin{array}{lc}
p_{\perp}^{2}+(2 n+1) g H & (D-2) \\
p_{\perp}^{2}+(2 n+3) g H & \text { multiplicity } \\
p_{\perp}^{2}+(2 n-1) g H & 1
\end{array} \quad(n=0,1, \cdots),\right.
$$

where the last term contains the Nielsen-Olesen unstable mode for $n=0$, i.e.,

$$
p_{\perp}^{2}-g H
$$


which becomes a tachyonic mode for small momenta $p_{\perp}^{2}<g H$.

where $W_{\Lambda}^{\prime}(\Theta)=\frac{d}{d \Theta} W_{\Lambda}(\Theta)$. Here we have introduced the wavefunction renormalization constants: $Z_{\Lambda}=Z_{\Lambda}^{\text {gluon }}, \tilde{Z}_{\Lambda}=Z_{\Lambda}^{\text {ghost }}$. In this derivation, we have adopted the truncation: neglecting the four-point interactions among the off-diagonal gluons and off-diagonal ghosts $-g^{2} \varepsilon^{a b 3} \varepsilon^{c d 3} i \bar{C}^{a} C^{d} A_{\mu}^{b} A_{\mu}^{c}$, which do not couple to the background field $V_{\mu}$.

The respective trace without the infrared regulator $R_{\Lambda}$ is easily obtained:

$$
\begin{aligned}
\Omega^{-1} \operatorname{Tr}\left[e^{-\tau\left(W_{\Lambda}^{\prime} Q\right)}\right] & =\frac{N g H}{(4 \pi)^{\frac{D}{2}}}\left(\tau W_{\Lambda}^{\prime}\right)^{1-\frac{D}{2}} \frac{2(D-2) e^{-\tau W_{\Lambda}^{\prime} g H}+2 e^{-3 \tau W_{\Lambda}^{\prime} g H}+2 e^{\tau W_{\Lambda}^{\prime} g H}}{1-e^{-2 \tau W_{\Lambda}^{\prime} g H}}, \\
\Omega^{-1} \operatorname{Tr}\left[e^{-\tau\left(-W_{\Lambda}^{\prime} \mathscr{D}^{2}\right)}\right] & =\frac{N g H}{(4 \pi)^{\frac{D}{2}}}\left(\tau W_{\Lambda}^{\prime}\right)^{1-\frac{D}{2}}\left[\frac{2 e^{-\tau W_{\Lambda}^{\prime} g H}}{1-e^{-2 \tau W_{\Lambda}^{\prime} g H}}\right], \\
\Omega^{-1} \operatorname{Tr}\left[e^{-\tau\left(-\tilde{Z}_{\Lambda} \mathscr{D}^{2}\right)}\right] & =\frac{N g H}{(4 \pi)^{\frac{D}{2}}}\left(\tau \tilde{Z}_{\Lambda}\right)^{1-\frac{D}{2}}\left[\frac{2 e^{-\tau \tilde{Z}_{\Lambda} g H}}{1-e^{-2 \tau \tilde{Z}_{\Lambda} g H}}\right],
\end{aligned}
$$

where $N=2$ for $S U(2)$.

In order to obtain the closed analytical form for the solution and to compare the FRG calculations with the loop calculations, we choose the momentum-independent infrared regular of the mass type:

$$
R_{\Lambda}^{\Phi}=Z_{\Lambda}^{\Phi} \Lambda^{2}
$$

where $Z_{\Lambda}^{\Phi}$ denotes the wave function normalization constant for the field $\Phi$. Moreover, we show later that the result is independent of the choice of the infrared regulator.

For the infrared regulator of the mass type, thus the flow equation reads

$$
\begin{aligned}
\partial_{t} \Gamma_{\Lambda}= & \frac{N g H}{(4 \pi)^{\frac{D}{2}}}\left\{\left(W_{\Lambda}^{\prime}\right)^{1-\frac{D}{2}}\left(2-\eta_{\Lambda}\right) Z_{\Lambda} \Lambda^{2} \int_{0}^{\infty} d \tau \tau^{1-\frac{D}{2}} e^{-\tau Z_{\Lambda} \Lambda^{2}} \frac{(D-2) e^{-\tau W_{\Lambda}^{\prime} g H}+e^{-3 \tau W_{\Lambda}^{\prime} g H}+e^{\tau W_{\Lambda}^{\prime} g H}}{1-e^{-2 \tau W_{\Lambda}^{\prime} g H}}\right. \\
& -\left(W_{\Lambda}^{\prime}\right)^{1-\frac{D}{2}}\left(2-\eta_{\Lambda}\right) Z_{\Lambda} \Lambda^{2} \int_{0}^{\infty} d \tau \tau^{1-\frac{D}{2}} e^{-\tau Z_{\Lambda} \Lambda^{2}} \frac{e^{-\tau W_{\Lambda}^{\prime} g H}}{1-e^{-2 \tau W_{\Lambda}^{\prime} g H}} \\
& +\alpha_{\Lambda}^{\frac{D}{2}-1}\left(2-\eta_{\Lambda}\right) Z_{\Lambda} \Lambda^{2} \int_{0}^{\infty} d \tau \tau^{1-\frac{D}{2}} e^{-\tau Z_{\Lambda} \Lambda^{2}} \frac{e^{-\tau \alpha_{\Lambda}^{-1} g H}}{1-e^{-2 \tau \alpha_{\Lambda}^{-1} g H}} \\
& \left.-\left(\tilde{Z}_{\Lambda}\right)^{1-\frac{D}{2}}\left(2-\tilde{\eta}_{\Lambda}\right) \tilde{Z}_{\Lambda} \Lambda^{2} \int_{0}^{\infty} d \tau \tau^{1-\frac{D}{2}} e^{-\tau \tilde{Z}_{\Lambda} \Lambda^{2}} \frac{2 e^{-\tau \tilde{Z}_{\Lambda} g H}}{1-e^{-2 \tau \tilde{Z}_{\Lambda} g H}}\right\}
\end{aligned}
$$

where we have introduced the anomalous dimensions:

$$
\eta_{\Lambda}:=-\partial_{t} \ln Z_{\Lambda}=-Z_{\Lambda}^{-1} \partial_{t} Z_{\Lambda}, \quad \tilde{\eta}_{\Lambda}:=-\partial_{t} \ln \tilde{Z}_{\Lambda}=-\tilde{Z}_{\Lambda}^{-1} \partial_{t} \tilde{Z}_{\Lambda}
$$

We find that the integral with respect to $\tau$ on the right-hand side of the flow equation is divergent at $D=4$ in the $\tau=0$ region which is an ultraviolet divergence. This divergence is independent of the infrared divergence coming from $\tau=\infty$ region due to the factor $e^{\tau W_{\Lambda}^{\prime} g H}$ for which the Nielsen-Olesen instability is responsible. This ultraviolet divergence is due to the fact that the momentum-independent infrared cutoff function of the mass type does not suppress the high-momenta. This aspect is a short-coming of the mass-type infrared regulator. However, the result will be true for any other choice of the infrared regulator, since the infrared regulator $R_{\Lambda}\left(p^{2}\right)$ 
is constructed so that any infrared cutoff function approaches the same asymptotic form as the mass-type one in the large $\Lambda$. See [11] for more details.

The ultraviolet divergence is removed by the standard method. Thus we arrive at the flow equation without the ultraviolet divergence:

$$
\begin{aligned}
\partial_{t} \Gamma_{\Lambda}= & \frac{N}{2} \frac{2 g H}{(4 \pi)^{2}}\left(-\ln \frac{2 g H}{4 \pi \mu^{2}}-\gamma\right)\left\{\left(W_{\Lambda}^{\prime}\right)^{-1}\left(2-\eta_{\Lambda}\right) Z_{\Lambda} \Lambda^{2}\right. \\
& \times\left[\zeta\left(0, \frac{1}{2}+\frac{Z_{\Lambda} \Lambda^{2}}{2 W_{\Lambda}^{\prime} g H}\right)+\zeta\left(0, \frac{3}{2}+\frac{Z_{\Lambda} \Lambda^{2}}{2 W_{\Lambda}^{\prime} g H}\right)+\zeta\left(0,-\frac{1}{2}+\frac{Z_{\Lambda} \Lambda^{2}}{2 W_{\Lambda}^{\prime} g H}\right)\right] \\
+ & \left.\alpha_{\Lambda}\left(2-\eta_{\Lambda}\right) Z_{\Lambda} \Lambda^{2} \zeta\left(0, \frac{1}{2}+\frac{Z_{\Lambda} \Lambda^{2}}{2 \alpha_{\Lambda}^{-1} g H}\right)-2\left(2-\tilde{\eta}_{\Lambda}\right) \Lambda^{2} \zeta\left(0, \frac{1}{2}+\frac{\Lambda^{2}}{2 g H}\right)\right\} \\
+ & \frac{N}{2} \frac{2 g H}{(4 \pi)^{2}}\left\{( W _ { \Lambda } ^ { \prime } ) ^ { - 1 } ( 2 - \eta _ { \Lambda } ) Z _ { \Lambda } \Lambda ^ { 2 } \left[\zeta^{(1,0)}\left(0, \frac{1}{2}+\frac{Z_{\Lambda} \Lambda^{2}}{2 W_{\Lambda}^{\prime} g H}\right)+\zeta^{(1,0)}\left(0, \frac{3}{2}+\frac{Z_{\Lambda} \Lambda^{2}}{2 W_{\Lambda}^{\prime} g H}\right)\right.\right. \\
& \left.+\zeta^{(1,0)}\left(0,-\frac{1}{2}+\frac{Z_{\Lambda} \Lambda^{2}}{2 W_{\Lambda}^{\prime} g H}\right)-2 \zeta\left(0, \frac{1}{2}+\frac{Z_{\Lambda} \Lambda^{2}}{2 W_{\Lambda}^{\prime} g H}\right)\right] \\
& \left.+\alpha_{\Lambda}\left(2-\eta_{\Lambda}\right) Z_{\Lambda} \Lambda^{2} \zeta^{(1,0)}\left(0, \frac{1}{2}+\frac{Z_{\Lambda} \Lambda^{2}}{2 \alpha_{\Lambda}^{-1} g H}\right)-2\left(2-\tilde{\eta}_{\Lambda}\right) \Lambda^{2} \zeta^{(1,0)}\left(0, \frac{1}{2}+\frac{\Lambda^{2}}{2 g H}\right)\right\} .
\end{aligned}
$$

Here we have introduced the generalized Riemann $\zeta$-function or the Hurwitz $\zeta$-function $\zeta(z, \lambda)$ defined by

$$
\zeta(z, \lambda):=\sum_{n=0}^{\infty} \frac{1}{(n+\lambda)^{z}}
$$

which has its integral representation

$$
\zeta(z, \lambda)=\frac{1}{\Gamma(z)} \int_{0}^{\infty} d s s^{z-1} \frac{e^{-\lambda s}}{1-e^{-s}}(\operatorname{Re} z>1, \operatorname{Re} \lambda>0) .
$$

Although the Hurwitz $\zeta$-function $\zeta(z, \lambda)$ is originally defined for $\operatorname{Re} z>1$, $\operatorname{Re} \lambda>0$, it can be analytically continued to other region in the complex $z$-plane as an analytic function.

\section{Absence of the Nielsen-Olesen instability}

For large $\Lambda$, we can take the approximation:

$$
\begin{aligned}
& \text { i) } W_{\Lambda}(\Theta)=\Theta \Rightarrow W_{\Lambda}^{\prime}(\Theta) \equiv 1 \Longleftrightarrow Z_{\Lambda} \equiv 1 \Rightarrow \eta_{\Lambda} \equiv 0, \\
& \text { ii) } \tilde{Z}_{\Lambda} \equiv 1 \Rightarrow \tilde{\eta}_{\Lambda} \equiv 0, \\
& \text { iii) } \alpha_{\Lambda} \equiv \alpha_{\Lambda_{\mathrm{UV}}}=\text { const. } \geq 0 .
\end{aligned}
$$

Then the flow equation can be cast into the total derivative form: $\partial_{t} \Gamma_{\Lambda}=\partial_{t}(\ldots)$. We take into account the fact that the effective average action $\Gamma_{\Lambda}$ at $\Lambda=\Lambda_{\mathrm{UV}}=\infty$ is given by the bare action for the classical chromomagnetic field background: $\Gamma_{\Lambda=\infty}=\frac{1}{4}\left(\mathscr{F}_{\mu \nu}^{A}[\mathscr{V}]\right)^{2}=\frac{1}{2} H^{2}$. Then an approximate 
solution is obtained by integrating the flow equation from $\Lambda=\Lambda_{\mathrm{UV}}=\infty$ to $\Lambda$ where $\tilde{V}_{\Lambda}(H)=0$ at $\Lambda=\infty$.

Thus, we arrive at the effective potential for large $\Lambda$, e.g., in the case of $\alpha_{\Lambda} \equiv 1$ :

$$
\begin{aligned}
V_{\Lambda}(H) & =\frac{1}{2} H^{2}+\frac{1}{16 \pi^{2}} \Lambda^{2}\left[\ln \frac{g H}{\mu^{2}}+\frac{1}{4}-C\right]-\frac{2}{16 \pi^{2}} g H \Lambda^{2} \ln \frac{\Lambda^{2}-g H}{\Lambda^{2}+g H} \\
& +\frac{1}{16 \pi^{2}} g^{2} H^{2}\left[\frac{11}{3} \ln \frac{g H}{\mu^{2}}+2 \ln \frac{\Lambda^{2}+g H}{g H}+2 \ln \frac{\Lambda^{2}-g H}{g H}\right. \\
& \left.-\frac{11}{3} C-4 \ln 2-\frac{1}{3}+8 \zeta^{(1,0)}\left(-1, \frac{1}{2}+\frac{\Lambda^{2}}{2 g H}\right)\right] .
\end{aligned}
$$

Note that $\zeta^{(1,0)}(-1, \lambda)$ is real-valued for $\lambda>0$.

For the large $\Lambda$ satisfying $\Lambda^{2} \geq g H, \tilde{V}_{\Lambda}(H)$ is real-valued and $V_{\Lambda}(H)$ has no imaginary part:

$$
\begin{gathered}
\operatorname{Im} V_{\Lambda}(H)=0 \text { for } \Lambda^{2} \geq g H, \\
\partial_{t} \operatorname{Im} V_{\Lambda}(H)=0 \text { for } \Lambda^{2} \geq g H .
\end{gathered}
$$

Therefore, the Nielsen-Olesen instability disappears for any value of $\Lambda$, in particular even at $\Lambda=0$ according to the above argument of the fixed point for the pure imaginary part of the flow equation.

For the small $\Lambda$ satisfying $\Lambda^{2}<g H$, however, the effective average potential $V_{\Lambda}(H)$ obtained above has the non-vanishing imaginary part:

$$
\operatorname{Im} V_{\Lambda}(H)=\frac{4}{16 \pi^{2}} g^{2} H^{2} \frac{\frac{\Lambda^{2}}{g H}-1}{2} \ln (-1) / i=\frac{1}{8 \pi} g H\left(g H-\Lambda^{2}\right) \text { for } \Lambda^{2}<g H,
$$

which yields the nontrivial flow of the imaginary part:

$$
\partial_{t} \operatorname{Im} V_{\Lambda}(H)=-\frac{1}{4 \pi} g H \Lambda^{2}<0 \text { for } \Lambda^{2}<g H .
$$

This is not a contradiction, since the approximate solution of $V_{\Lambda}(H)$ obtained above is not considered to be valid in the small $\Lambda$ region; $\Lambda^{2}<g H$. In fact, the derivative $\partial_{t} \operatorname{Im} V_{\Lambda}(H)$ has the discontinuity at $\Lambda^{2}=g H$. The effective potentials obtained above reproduce the Nielsen-Olesen result by putting $\Lambda=0$.

\section{5. gluon mass generation and vacuum condensations}

The above approximate solution (4.2) eventually has the imaginary part and hence cannot be used in the limit $\Lambda \rightarrow 0$. As will be shown in this section, however, the approximate solution obtained in the same type of approximations has the limit $\Lambda \rightarrow 0$ without developing the imaginary part, if the effects of mass generation are incorporated into the analysis. Such mass generation is expected to occur, as established in the numerical simulations on the lattice [17] 18].

We introduce the mixed composite operators of gluons and ghosts: For $S U(2)$,

$$
\mathscr{O}=\frac{1}{2} A_{\mu}^{a} A^{\mu a}+\alpha i \bar{C}^{a} C^{a} \quad(a=1,2) .
$$


and study the mass generation for the off-diagonal gluons (and ghosts), originating from the dimensiontwo condensation $\langle\mathscr{O}\rangle$. It is shown [13] that the dimension-two condensation $\langle\mathscr{O}\rangle$ is BRST invariant in the modified MA gauge [22] defined by the GF+FP term, i.e., the $\operatorname{OSp}(D, 2)$-invariant form:

$$
\mathscr{L}_{\mathrm{GF}+\mathrm{FP}}^{\mathrm{MA}}=i \boldsymbol{\delta} \overline{\boldsymbol{\delta}}\left(\frac{1}{2} A_{\mu}^{a} A_{\mu}^{a}+\frac{\alpha}{2} i \bar{C}^{a} C^{a}\right)
$$

where $\boldsymbol{\delta}$ and $\overline{\boldsymbol{\delta}}$ are respectively the BRST and anti-BRST transformations.

According to [23], we introduce a new field $\phi$ which is an auxiliary field with no kinetic term represented by the Lagrangian density:

$$
\mathscr{L}_{\phi}=\frac{1}{2}(\phi+G \mathscr{O})^{\dagger} G^{-1}(\phi+G \mathscr{O})=\frac{1}{2} \phi^{\dagger} G^{-1} \phi+\phi^{\dagger} \mathscr{O}+\frac{1}{2} \mathscr{O}^{\dagger} G \mathscr{O}
$$

by inserting the unity: $1=\int D \phi e^{-\int d^{D} x \mathscr{L}_{\phi}}$, in the path-integral measure. We observe:

- From the first term $\frac{1}{2} \phi^{\dagger} G^{-1} \phi$, we observe that $G$ represents the effective propagator of the collective field $\phi$, i.e., two-gluon bound state propagator.

- The second term $\phi^{\dagger} \mathscr{O}$ yields the cubic interactions $\phi A A$ (and $\phi \bar{C} C$ ) for the operator $\mathscr{O}$ quadratic in the off-diagonal gluons (and ghosts).

- The third term $\frac{1}{2} \mathscr{O}^{\dagger} G \mathscr{O}$ involving only the fundamental fields has the form of an exchange of $\phi$ in the tree approximation.

By including $\mathscr{L}_{\phi}$, the two-point functions $\Gamma_{\Lambda}^{(2)}$ are modified as

$$
\left(\Gamma_{\Lambda}^{(2)}\right)_{A_{\mu}^{a} A_{\nu}^{b}}=W_{\Lambda}^{\prime} Q_{\mu \nu}^{a b}+\varphi \delta_{\mu \nu} \delta^{a b}, \quad\left(\Gamma_{\Lambda}^{(2)}\right)_{\bar{C}^{a} C^{b}}=-\tilde{Z}_{\Lambda}\left(\mathscr{D}^{2}\right)^{a b}+\alpha_{\Lambda} \varphi \delta^{a b},
$$

where $\varphi=\langle\phi\rangle$. Here we have adopted the truncation: neglecting the four-point interactions among the off-diagonal gluons and off-diagonal ghosts.

We use the infrared regulator of the mass type and the same approximations for $W_{\Lambda}, \tilde{Z}_{\Lambda}$ and $\alpha_{\Lambda}$ as those adopted in the previous case. Then we obtain the effective average potential $V_{\Lambda}(H, \varphi)$ describing the chromomagnetic condensation and dynamical mass generation simultaneously. We consider the simplest case of $\alpha_{\Lambda} \equiv 1$ to clarify the qualitative feature (see [14] for a physical meaning of the dimension-two condensate in this gauge). In this case, the effective potential is given by

$$
\begin{aligned}
V_{\Lambda}(H, \varphi)= & \frac{1}{2 g_{\Lambda}^{2}} H^{2}+\frac{1}{2 G_{\Lambda}} \varphi^{2}+\tilde{V}_{\Lambda}(H, \varphi) \\
\tilde{V}_{\Lambda}(H, \varphi)= & -\frac{1}{4 \pi^{2}} H^{2}\left(\ln \frac{H}{\mu^{2}}-C\right)\left[\zeta\left(-1, \frac{3}{2}+\frac{X}{2 H}\right)+\zeta\left(-1,-\frac{1}{2}+\frac{X}{2 H}\right)\right] \\
& +\frac{1}{4 \pi^{2}} H^{2}\left[\zeta^{(1,0)}\left(-1, \frac{3}{2}+\frac{X}{2 H}\right)+\zeta^{(1,0)}\left(-1,-\frac{1}{2}+\frac{X}{2 H}\right)-2 \zeta\left(-1, \frac{1}{2}+\frac{X}{2 H}\right)\right], \\
X:= & \varphi+\Lambda^{2} .
\end{aligned}
$$


Here we have rescaled $H$ as $H \rightarrow \frac{1}{g} H$ for later convenience so that the quantum parts $\tilde{V}_{\Lambda}$ does not include the $g$ dependence. We find that $\tilde{V}_{\Lambda}(H, \varphi)$ is obtained form $\tilde{V}_{\Lambda}(H)=\tilde{V}_{\Lambda}(H, \varphi=0)$ by shifting the variable $\Lambda^{2} \rightarrow \Lambda^{2}+\varphi$ :

$$
\tilde{V}_{\Lambda}(H, \varphi)=\left.\tilde{V}_{\Lambda}(H, \varphi=0)\right|_{\Lambda^{2} \rightarrow X}=\left.\tilde{V}_{\Lambda}(H)\right|_{\Lambda^{2} \rightarrow X} .
$$

The real-valuedness condition for $V_{\Lambda}$ is replaced by $X-H>0$, or $H<X:=\varphi+\Lambda^{2}$. In other words, the stability excludes the region: $H \geq X:=\varphi+\Lambda^{2}$. Therefore, we define the allowed region for stability:

$$
\mathscr{R}_{\Lambda}=\left\{(H, \varphi) ; H<X:=\varphi+\Lambda^{2}, H \geq 0, \varphi>0\right\} .
$$

which is a region below the straight line $H=X$ with the slope 1 and intercept $\Lambda^{2}$.

$V_{\Lambda}(H, \varphi)$ can be made real-valued by taking sufficiently large $\Lambda$, as in the case of $V_{\Lambda}(H)$. In the absence of $\varphi$, this argument for eliminating the imaginary part does not work in the small $\Lambda$ region in which the inequality $H>\Lambda^{2}$ is satisfied. This shortcoming is avoided by including $\varphi$. In fact, the allowed region for stability $\mathscr{R}_{\Lambda}$ becomes narrower for lower value of $\Lambda$, but survives even in the limit $\Lambda \rightarrow 0$. Hence, the $H$ axis or $\varphi=0$ is excluded in the limit $\Lambda \rightarrow 0$.

The running coupling $g_{\Lambda}$ is monotonically increasing in decreasing $\Lambda$. Therefore, the tree term $\frac{1}{2} g_{\Lambda}^{-2} H^{2}$ also becomes negligible for small enough $\Lambda$.

We can write down the flow equation for $G_{\Lambda}$. Solving it, we find that $G_{\Lambda}^{-1}$ monotonically decreases as $\Lambda$ decreases. Therefore, the effect of the tree term $\frac{1}{2} G_{\Lambda}^{-1} \varphi^{2}$ becomes more and more negligible for smaller $\Lambda$. In fact, the increasing of $G_{\Lambda}$ in decreasing $\Lambda$ is reasonable, since the bound state propagator $G_{\Lambda}(s)$ will approach the structure with a pole-like dependence on $s$ for small enough $\Lambda$ [24]. Therefore, the details of the behavior of $G_{\Lambda}$ does not change the following result qualitatively.

Thus the existence and location of the minimum can be dominantly determined by the quantum part $\tilde{V}(H, \varphi)$. In view of these, we have looked for the minimum of $\tilde{V}_{\Lambda}(H, \varphi)$ in the region $\mathscr{R}_{\Lambda}$.

\section{Conclusion and discussion}

We have shown that the Nielsen-Olesen instability of the Savvidy vacuum with homogeneous chromomagnetic condensation is avoided in the framework of the FRG. Actually, we have shown that the imaginary part of the effective average action vanishes at sufficiently large infrared cutoff $\Lambda$, and this property can survive at $\Lambda=0$. This behavior can be understood as a fixed point solution of the flow equation for the complex-valued effective average action. Therefore, the Nielsen-Olesen instability is an artifact of the loop calculation in the perturbation theory.

Moreover, we have discussed the physical mechanism for keeping the stability for smaller $\Lambda$ : the stability is maintained even for small $\Lambda$ once the mass generation occurs for the off-diagonal gluons (and off-diagonal ghosts). See [25] for the related works.

The comparison of our result for the effective potential with that of [10] suggests that (i) $H \neq 0$ and $\varphi \neq 0$ is realized in the Yang-Mills vacuum. Using these solutions [9], moreover, we are able to discuss the possible relationship between the stability and the scaling/decoupling solutions which are recently claimed to be the true infrared solutions in the deep infrared region realizing quark and gluon confinement. These issues will be further discussed in future works. 


\section{References}

[1] Y. Nambu, Phys. Rev. D10, 4262-4268 (1974).

G. 't Hooft, in: High Energy Physics, edited by A. Zichichi (Editorice Compositori, Bologna, 1975).

S. Mandelstam, Phys. Report23, 245-249 (1976).

A.M. Polyakov, Phys. Lett. B59, 82-84 (1975). Nucl. Phys. B120, 429-458 (1977).

[2] G.K. Savvidy, Phys. Lett. B 71, 133-134 (1977).

[3] N.K. Nielsen and P. Olesen, Nucl. Phys. B 144, 376-396 (1978).

[4] N.K. Nielsen, P. Olesen, Phys. Lett. B79, 304-308 (1978).

J. Ambjorn, N.K. Nielsen, and P. Olesen, Nucl. Phys. B152, 75-96 (1979).

H. B. Nielsen, M. Ninomiya, Nucl.Phys. B156, 1-28 (1979).

H. B. Nielsen and P. Olesen, Nucl. Phys. B160, 380-396 (1979)

J. Ambjorn and P. Olesen, Nucl. Phys. B170, 60-76 (1980).

[5] C. Wetterich, Phys. Lett. B301, 90-94 (1993).

[6] M. Reuter and C. Wetterich, Nucl. Phys. B417, 181-214 (1994).

[7] M. Reuter and C. Wetterich, Phys. Rev. D56, 7893-7916 (1997).

[8] D. F. Litim and J. M. Pawlowski, Phys. Rev. D66, 025030 (2002).

[9] H. Gies, Phys. Rev. D66, 025006 (2002).

[10] A. Eichhorn, H. Gies, and J. M. Pawlowski, Phys. Rev. D83, 045014 (2011), Erratum-ibid. D83, 069903 (2011).

[11] K.-I. Kondo, e-Print: arXiv:1309.2337 [hep-th].

[12] F.V. Gubarev, L. Stodolsky and V.I. Zakharov, Phys. Rev. Lett. 86, 2220-2222 (2001). F.V. Gubarev and V.I. Zakharov, Phys. Lett. B501, 28-36 (2001).

[13] K.-I. Kondo, Phys. Lett. B514, 335(2001).

[14] K.-I. Kondo, Phys. Lett. B572, 210(2003).

[15] K.-I. Kondo, T. Murakami, T. Shinohara and T. Imai, Phys. Rev. D65, 085034 (2002).

[16] G. 't Hooft, Nucl.Phys. B190 [FS3], 455-478 (1981).

[17] K. Amemiya and H. Suganuma, Phys. Rev. D60, 114509 (1999).

[18] V.G. Bornyakov, M.N. Chernodub, F.V. Gubarev, S.M. Morozov and M.I. Polikarpov, Phys. Lett. B559, 214-222 (2003).

[19] T. Mendes, A. Cucchieri and A. Mihara, AIP Conf. Proc.892, 203(2007).

[20] K.-I. Kondo, Phys. Rev. D57, 7467-7487 (1998).

[21] K.-I. Kondo, Phys. Rev. D82, 065024 (2010).

[22] K.-I. Kondo, Phys. Rev. D58, 105019 (1998).

[23] U. Ellwanger and C. Wetterich, Nucl. Phys. B423, 137-170 (1994).

[24] U. Ellwanger, Z.Phys. C62, 503-510 (1994)

[25] K.-I. Kondo, Phys. Lett. B 600, 287-296 (2004).

K.-I. Kondo, T. Murakami and T. Shinohara, Eur. Phys. J. C 42, 475-481 (2005).

K.-I. Kondo, T. Murakami and T. Shinohara, Prog. Theor. Phys. 115, 201-216 (2006).

K.-I. Kondo, Phys. Rev. D74, 125003 (2006). 